\title{
Ionization-stratification in Wolf-Rayet atmospheres
}

\author{
Krzysztof Rochowicz \\ Centre for Astronomy, N. Copernicus University, Torun, Poland
}

\begin{abstract}
We summarize an analysis of low-resolution IUE archival data for galactic and LMC Wolf-Rayet stars. We examine the correlation between ionization potential with line-width (FWHM) constituting a sample of stars showing a well-defined UV ionization structure.
\end{abstract}

\section{Introduction}

Ionization-stratification of Wolf-Rayet winds has been recognized spectroscopically by Beals (1929) based on an observed inverse correlation of emission line width with ionization potential for both WN and WC stars. These results implied a decrease in the degree of ionization with increasing radius.

A relationship has also been noted between excitation potential and linewidth by Willis (1982). The predictions of ionization- and excitation-stratification made by the standard model of Hillier (1989) have been confirmed observationally once more by Dalton et al. (1995).

In an accelerated outflow the stratification is observable through the Doppler effect as an inverse correlation of IP with line-width (FWHM). However, not only the average line-widths of different ions, but also the line-widths of one series of e.g., He II show stratification. The evidence of both effects on WR UV spectra was demonstrated by Rochowicz (1997).

\section{Observational material}

The ultraviolet spectra of $94 \mathrm{WR}$ stars were obtained from the ESA IUE archive. We have tried to select as many single stars as possible in the Galaxy and the LMC to get the complete, flux-calibrated spectra. Together 56 objects in the Galaxy and 38 in the LMC were found, for which at least single SWP and LWR (or LWP) low-resolution images are accessible - note that this is about one third of the number of known galactic and LMC WR stars. The set of stars we study is representative in spectral subtype and brightness (it is almost complete to $V=12$ for Galactic stars). See Niedzielski \& Rochowicz (1994) for the list of program stars and for the details of reduction. The original data (FWHM) are available at the CDS via anonymous ftp 130.79.128.5.

The aim of our project was to analyze UV Wolf-Rayet stars spectra. So far the spectra were used for e.g., :

- searching for correlations among the strength and widths of emission lines of different ions (Niedzielski \& Rochowicz 1994) 
- spectral classification in the ultraviolet (Rochowicz 1995)

- determining terminal velocities (Rochowicz \& Niedzielski 1995)

- deriving fundamental physical parameters (Rochowicz 1996)

\section{Discussion}

Ionization-stratification can be traced on the traditional plot of half-width velocity versus IP - the mean width of the lower ion lines is wider than the mean width of the next ion lines, as is expected according to the increasing IPs of these lines (closer to the stellar core).

The least-squares fit to the points gives a relation of the form:

$$
v=v_{0}+g \times I P
$$

where $v_{0}$ is FWHM at zero IP and $g$ is gradient of the fit.

Quantitative analysis of traditional plots of FWHM vs. IP for a large sample of stars was performed. We included on the average about 20 emission lines in each case. The WC stars, in spite of their complicated spectra, seem to form a more uniform group; we note a large scatter for WN stars. Altogether 36 objects in the Galaxy and 24 in the LMC clearly show ionization structure. Details will be published elsewhere.

There does not appear to be a trend connecting the gradient $(g)$ or FWHM at zero IP $\left(v_{0}\right)$, with either spectral subtype or terminal wind velocity.

The information available from stratification studies plays a crucial role in the development of our knowledge of wind dynamics and variability of the WR stars.

Acknowledgments. This research was supported by KBN grant No.2 PO3D 00512 .

\section{References}

Beals, C.S. 1929, MNRAS 90, 202

Dalton, M.J., Crowther, P.A., Willis, A.J. 1995, in: K.A. van der Hucht \& P.M. Williams (eds.), Wolf-Rayet Stars: Binaries, Colliding Winds, Evolution, Proc. IAU Symp. No 163 (Dordrecht: Kluwer), p. 154

Hillier, D.J. 1989, ApJ 347, 392

Niedzielski, A., Rochowicz, K. 1994, A\&AS 108, 669

Rochowicz, K., Niedzielski A. 1995, Acta Astron. 45, 307

Rochowicz, K. 1995, in: K.A. van der Hucht \& P.M. Williams (eds.), Wolf-Rayet Stars: Binaries, Colliding Winds, Evolution, Proc. IAU Symp. No 163 (Dordrecht: Kluwer), p. 50

Rochowicz, K. 1996, in: I. Lundström \& B. Stenholm (eds.), Planetary Nebulas with Wolf-Rayet Type Nuclei, ApSS 238, 71

Rochowicz, K. 1997, Acta Astron. 47, 381

Willis, A.J. 1982, MNRAS 198, 897 